\title{
BMJ Open Measures of central sensitisation and their measurement properties in the adult musculoskeletal trauma population: a protocol for a systematic review and data synthesis
}

Nicola Middlebrook, Alison B Rushton, ${ }^{\odot}$ Nicola R Heneghan, ${ }^{\odot}$ Deborah Falla ${ }^{\odot}$

To cite: Middlebrook N, Rushton AB, Heneghan NR, et al. Measures of central sensitisation and their measurement properties in the adult musculoskeletal trauma population: a protocol for a systematic review and data synthesis. BMJ Open 2019;9:e023204. doi:10.1136/ bmjopen-2018-023204

- Prepublication history and additional material for this paper are available online. To view these files, please visit the journal online (http://dx.doi org/10.1136/bmjopen-2018023204).

Received 26 March 2018 Revised 12 December 2018 Accepted 21 January 2019

A) Check for updates

(C) Author(s) (or their employer(s)) 2019. Re-use permitted under CC BY-NC. No commercial re-use. See rights and permissions. Published by BMJ.

Centre of Precision

Rehabilitation for Spinal Pain (CPR Spine), School of Sport, Exercise and Rehabilitation Sciences, College of Life and Environmental Sciences, University of Birmingham, Birmingham, UK

Correspondence to Professor Deborah Falla; d.falla@bham.ac.uk

\section{ABSTRACT}

Introduction Pain following musculoskeletal trauma is common with poor outcomes and disability well documented. Pain is complex in nature and can include the four primary mechanisms of pain: nociceptive, neuropathic, inflammatory and central sensitisation (CS). CS can be measured in multiple ways; however, no systematic review has evaluated the measurement properties of such measures in the musculoskeletal trauma population. This systematic review aims to evaluate the measurement properties of current measures of CS in this population.

Methods/analysis This protocol is informed and reported in line with the Preferred Reporting Items for Systematic Reviews and Meta-Analysis-P. MEDLINE, EMBASE, CINAHL, ZETOC, Web of Science, PubMed and Google Scholar as well as key journals and grey literature will be searched in two stages to (1) identify what measures are being used to assess CS in this population and (2) evaluate the measurement properties of the identified measures. Two independent reviewers will conduct the search, extract the data, assess risk of bias for included studies and assess overall quality. The Consensus-based Standards for the selection of Health Measurement Instruments Risk of Bias Checklist and a modified Grading of Recommendations, Assessment, Development and Evaluation guidelines will be used. Meta-analysis will be conducted if deemed appropriate. Alternatively, a narrative synthesis will be conducted and summarised per measurement property per outcome measure.

Ethics and dissemination This review will aid clinicians in using the most appropriate tool for assessing central sensitisation in this population and is the first step towards a more standardised approach in pain assessment. The results of this study will be submitted to a peer reviewed journal and presented at conferences. PROSPERO registrationnumber CRD42018091531.

\section{INTRODUCTION}

Pain is a common occurrence following musculoskeletal trauma and is routinely assessed in both research and clinical practice. Many methods exist for assessing pain
Strengths and limitations of this study

- This is the first systematic review to evaluate measurement properties of central sensitisation (CS) outcome measures in the musculoskeletal trauma population.

- This study utilises the new Consensus-based Standards for the selection of Health Measurement Instruments Risk of Bias tool for systematic reviews to evaluate methodological quality.

- While assessing other pain outcome measures in this population would be beneficial, this study is limited to measures of CS only.

and commonly include patient reported outcome measures (PROMs) such as the Numerical Rating Scale or the McGill Pain Questionnaire. ${ }^{1}$ Many of these measures have established measurement properties and are recommended as outcome measures for clinical trials in chronic pain populations. ${ }^{1}$

However, these methods are limited to being patient reported and often focus only on pain intensity. ${ }^{2}$ While this is relevant and focuses on patient perception and impact of pain, this does not identify the neurophysiological mechanisms driving pain perception. ${ }^{3}$ Identifying the underlying pain mechanisms for individual patients has the potential to allow more targeted treatments resulting in better outcomes. ${ }^{4}$ Furthermore, measures that incorporate the four domains (body function, activities and participation, body structure and environmental factors) of the International Classification of Functioning, Disability and Health allow a more holistic approach in assessing patients. ${ }^{5}$

Four main pain mechanisms have been described: nociceptive (activation of nociceptors from actual or threatened tissue damage), neuropathic (damage, lesion or 
disease of the somatosensory nervous system), inflammatory (nociceptors activated by inflammatory mediators due to tissue damage) and central sensitisation (CS) ${ }^{46} \mathrm{CS}$ can be described when the central nervous system adapts and amplifies pain, often when tissue healing has taken place and no further pathology or disease process can be identified. ${ }^{4} \mathrm{CS}$ results from increased excitability of central nociceptive neurons in the cortex, brain stem, trigeminal nucleus and spinal cord. ${ }^{8}$ A number of mechanisms have been implicated in long-term CS, including cortical reorganisation and maladaptive neuroplasticity, alterations in neurochemistry, loss of inhibitory neurons, alerted glial activity, dysfunction of endogenous pain control mechanisms, alterations in grey-matter volume and altered structural integrity and connectivity of white matter. ${ }^{8}$ This results in general hypersensitivity of the somatosensory system, presenting with symptoms such as widespread pain, tactile allodynia, secondary hyperalgesia and a heightened response to non-noxious stimuli. ${ }^{7}$ With multiple presentations and features, the underlying pathophysiological cause of CS is debated within the literature $;{ }^{37}$ therefore, CS is often regarded as a concept rather than a true diagnostic label.

Features of CS have been identified in many chronic pain disorders such as osteoarthritis, fibromyalgia, chronic whiplash-associated disorders and low back pain. ${ }^{79-11}$ Patients presenting with symptoms of CS typically show higher pain ratings, ${ }^{12}$ greater disability, ${ }^{13}$ are more likely to develop future musculoskeletal conditions $^{14}$ and show poorer outcomes and increased likelihood of developing chronic pain. ${ }^{15}$ Features of CS have also been observed within the early stages of acute injury, for example, following a whiplash trauma, and have been shown to be predictive of poorer outcome in this population. $^{1617}$

Within musculoskeletal trauma, poor outcomes and disability are well documented, ${ }^{18-20}$ with the question of why some people fully recover when others do not still to be answered. Recent studies recognise this issue within musculoskeletal trauma and are moving towards a more mechanistic approach in assessing patients to optimise recovery. ${ }^{21}$ While not all musculoskeletal trauma patients will present with features of CS, and be the main cause of poor outcome, it should not be ruled out as a cause or predictor of poor recovery and should be included within assessment of pain.

Various methods exist to assess CS including quantitative sensory testing (QST) ${ }^{3}$ which aims to assess the function of the somatosensory system using various methods such as thermal and pressure pain thresholds, ${ }^{22}{ }^{23}$ pain drawings where more widespread symptoms can be assessed $^{3}$ and PROMs such as the Central Sensitisation Inventory ${ }^{24}$ which incorporate common symptoms of CS. The measurement properties of these measures have been reported; however, the population sample is often healthy volunteers ${ }^{25}$ or an alternative population group ${ }^{22}$ which questions the value of these measures within a specific population such as musculoskeletal trauma. ${ }^{26}$
Established measurement properties for outcome measures are needed to avoid any potential bias and to be confident in findings when making recommendations. ${ }^{27}$ To inform and improve the selection of outcome measures, the Consensus-based Standards for the selection of Health Measurement instruments (COSMIN) initiative developed a consensus-based taxonomy of measurement properties with three main domains identified — reliability, validity and responsiveness- all having further subgroups of measurement properties. ${ }^{28}$ Furthermore, the COSMIN Checklist to assess methodological quality is a well-used tool both in systematic reviews and design and reporting of a study on measurement properties. A recent updated tool has been introduced specifically to use in systematic reviews. ${ }^{29} 30$

Currently, within musculoskeletal trauma, a vast range of measures are used to assess CS, highlighting a lack of consensus or gold standard measurement. ${ }^{31} 32$ Previous systematic reviews investigating musculoskeletal pain and injury have highlighted the lack of consensus on the optimal measure of $\mathrm{CS},{ }^{31}{ }^{33}$ all finding poor or limited methodological quality within the studies. ${ }^{334}$ All reviews have used different tools to measure quality, with meta-analysis not possible due to the heterogeneity of included studies, making conclusions and clinical implications difficult. A systematic review is needed to synthesise and evaluate current practice in assessing CS to allow a more standardised approach, as well as being the first systematic review specifically investigating the musculoskeletal trauma population. Specifically, the primary aim of this systematic review is to investigate if current outcome measures of CS have established measurement properties in the musculoskeletal trauma population, with a secondary aim to inform and summarise what measures are currently being used in this population.

\section{METHODS}

This systematic review has been designed following scoping searches of the available literature and is reported in accordance to Preferred Reporting Items for Systematic Reviews and Meta-Analysis-P guidelines (online supplementary file 1). ${ }^{35}$

\section{Eligibility criteria \\ Inclusion criteria \\ Population}

Any study that examined an adult population (aged $\geq 16$ years) who experienced any type of musculoskeletal trauma. For the purpose of this review, musculoskeletal trauma will be defined as any musculoskeletal structure for example, bones, joints, ligaments, tendons and muscles that surround these structures involved in a traumatic injury. ${ }^{36}$ Examples of a traumatic injury include road traffic collisions, falls and gunshot and stab wounds. Studies that specifically investigate a population such as people following a whiplash injury will be included as this is deemed musculoskeletal in nature despite this 
population often being segregated and investigated individually. This is to ensure that the musculoskeletal trauma population is represented fully in this review. For studies with mixed aetiology, majority of the sample must be musculoskeletal in nature $(\geq 90 \%)$, a figure previously used in systematic reviews in this population. ${ }^{36}$

\section{Outcome measures}

Any study that evaluates CS using a specific outcome measure will be included. With no gold standard measure for CS, this makes defining eligibility criteria challenging. In order to establish robust criteria, the following criteria are based on current consensus in the literature and previous systematic reviews in the field. ${ }^{4-9} 3132$

Studies will be included if one of the following is highlighted:

1. Reference to sensitisation of the central nervous system using terms such as CS, central sensitivity, central pain modulation and widespread pain.

2. Reference to sensitisation symptoms specifically around sensory changes such as secondary hyperalgesia and tactile allodynia-symptoms well documented as indicators of CS. ${ }^{7}$

Outcome measures being defined as any one of the following:

1. Patient-reported measures or questionnaires designed specifically to assess CS.

2. Performance-based measures designed to assess aspects of CS, an example being QST - a term encompassing multiple sensory testing methods, which has been established as a good measure of features of CS. ${ }^{31}$

3. Measures that are evaluating symptoms of CS, for example, widespread pain using pain drawings.

\section{Measurement properties}

Any study that has evaluated one or more measurement properties will be included in this study. This will include all measurement properties included in the three main domains (reliability, validity and responsiveness) of the COSMIN Taxonomy. ${ }^{27}$ Definitions of measurement properties will be in line with the COSMIN taxonomy and are summarised in online supplementary file 2 .

\section{Study design}

Any study design apart from case studies and previous literature reviews including systematic reviews and meta-analyses will be included in this study — this includes randomised controlled trials, cohort studies, and observational studies.

\section{Timing and setting}

No restriction on length of study will be used in this review, and all-time points will be included, for example, from point of injury onwards. No restriction on setting will be used in this review.

\section{Exclusion criteria}

Any study where the primary injury is a traumatic brain injury, burns or neurological injury such as spinal cord injuries will be excluded. These subgroups of patients will be excluded due to various other specific measures being developed for these patients, for example, neurological-specific PROMs validated in this population, ${ }^{37}$ together with other factors such as reduced consciousness and pain not a primary factor in recovery, for example, brain injury. ${ }^{38}$ Papers that are not written in English will be excluded.

\section{Information sources}

A search strategy has been developed using medical subject headings, where available, and relevant text words relating to musculoskeletal trauma, CS and measurement properties. Databases to be encompassed in the search will include MEDLINE (OVID interface), EMBASE (OVID interface), CINAHL (EBSCO interface), ZETOC, Web of Science, PubMed and Google Scholar from inception.

Hand searching of key journals such as Musculoskeletal Science and Practice, PAIN, European Journal of Pain, The Journal of Pain and The Clinical Journal of Pain as well as contacting relevant leading researchers in the field will be conducted. Grey literature searching will include British National bibliography for report literature, openGrey, dissertation abstracts and EThOS.

\section{Search strategy}

Two independent reviewers will complete the search and identify potential studies to be included in the review. No study design, date or language limits will be used during the search, to ensure that no relevant studies are missed. An initial MEDLINE search strategy will be developed and then adapted with relevant syntax and subject headings for other databases. Recommended search filters specifically designed for retrieving articles on measurement properties will be used where appropriate. ${ }^{39} 40$

The search will take place in two stages:

1. Initial search to identify the outcome measures used in the musculoskeletal trauma population.

2. Secondary search identifying studies assessing measurement properties. This secondary search will focus on the outcome measures that were identified in the first search only.

In the event that there are a limited number of studies to evaluate measurement properties (four or less) in the specific population of adult musculoskeletal trauma, the secondary search will be widened to general musculoskeletal conditions such as osteoarthritis and fibromyalgia or healthy subjects, but findings will be evaluated and synthesised separately. Healthy subjects will be included as well as musculoskeletal conditions in order to capture studies that have evaluated measurement properties such as reliability, which can then inform future studies in a symptomatic population. An example of the search strategy for both stages is summarised in online supplementary file 3 .

\section{Data management}

All search results will be uploaded and stored on Endnote Version X8 (Clarivate Analytics) software 
programme. This will allow any duplicates to be identified and removed. Abstracts and full texts will be stored on Endnote. Screening questions and forms will be developed based on inclusion/exclusion criteria for the title and abstract screening and full-text retrieval.

\section{Study selection}

Two reviewers will independently screen titles/abstracts against the prespecified inclusion/exclusion criteria and subcategorise into include/exclude/unsure. ${ }^{41}$ Full texts will then be obtained for all articles that appear to meet the inclusion criteria. If there is uncertainty in inclusion criteria for a specific paper, the full text will be retrieved for further clarification. ${ }^{42}$ Authors will be contacted for additional information if required. Screening of full texts will be conducted in the same manner using the predefined criteria and conducted independently.

At both stages, articles will be included if both authors agree that the eligibility criteria are met. Any disagreement between the two reviewers will be first discussed in a consensus meeting between the reviewers, and if needed an independent reviewer sought if no agreement can be made. Information on excluded studies and reasons for exclusion will be collated and reported.

\section{Data collection process}

A standardised form developed for this review will be used to extract information from included studies. Both reviewers will independently extract information, with one reviewer then collating the information. Any disagreements between the reviewers will be discussed and/or reviewed by a third reviewer. Prior to data collection, a pilot of the data collection form will be conducted to ensure all relevant information is collected, and updated if needed.

\section{Data items}

The items to be extracted from included studies are summarised in table 1. Authors will be contacted for

\begin{tabular}{ll}
\hline $\begin{array}{l}\text { Table } 1 \text { Summary of items to be extracted from included } \\
\text { studies }\end{array}$ & Data items \\
\hline Content & Authors, year of publication \\
\hline $\begin{array}{l}\text { General study Information } \\
\text { Study characteristics }\end{array}$ & $\begin{array}{l}\text { Sample size, study design, } \\
\text { measurement time points, } \\
\text { country }\end{array}$ \\
\hline Patient characteristics & $\begin{array}{l}\text { Age, gender, type of trauma, } \\
\text { mechanism of trauma, duration } \\
\text { of symptoms }\end{array}$ \\
\hline Outcome measures & $\begin{array}{l}\text { CS outcome measures } \\
\text { Other outcome measures used } \\
\text { Measurement properties } \\
\text { assessing statistical methods } \\
\text { used and results }\end{array}$ \\
\hline
\end{tabular}

CS, central sensitisation. clarification and any missing data if needed. In the event of two or more papers being identified from the same study, authors will be contacted for further information to ensure the data are not duplicated in the review.

\section{Outcomes and prioritisation}

As no gold standard method exists for CS, no primary outcome measure is identified for this review.

\section{Risk of bias in individual studies}

The COSMIN Risk of Bias Checklist for systematic reviews will be used for risk of bias and methodological quality in individual studies. ${ }^{29}$ Revised from the original COSMIN Checklist, this new tool is specifically designed to evaluate studies on measurement properties in systematic reviews. The new checklist includes standards for each measurement property for both design and preferred statistical methods, and rates each study as either very good, adequate, doubtful and inadequate quality. ${ }^{29} 40$ Although currently no literature exists on the measurement properties of the new tool, the previous COSMIN Checklist demonstrated adequate reliability, ${ }^{43}$ a basis from which this new tool has subsequently been developed. As the COSMIN Risk of Bias Checklist was originally developed for PROMs, as with previous COSMIN tools, it can be adapted for other measures that are not PROMs, which is recommended by the COSMIN group. ${ }^{40}$ As with the screening process, two independent reviewers will assess and score the risk of bias for all included studies. Any disagreements between the two reviewers will be discussed in a consensus meeting. If no agreement can be made, again a third reviewer will be consulted. The percentage of agreement between the two reviewers will be reported in the results section of the final review.

\section{Data synthesis}

Following scoping searches of current available literature together with the heterogeneity of the population, pooling of data may not be possible; however, it will not be ruled out and will be assessed prior to synthesis and conducted if possible. If deemed not possible, a narrative synthesis will be conducted. Synthesis of results will follow recommendations from the COSMIN guidelines for systematic reviews. ${ }^{40}$ Individual studies will be assessed for methodological quality and rated against the predefined criteria for good measurement properties (online supplementary file 2). ${ }^{40}$ Evidence will be pooled or summarised per measurement property per outcome measure with the overall result rated again against the criteria for good measurement properties. The overall quality of evidence will then be assessed using a modified Grading of Recommendations Assessment, Development and Evaluation (GRADE) approach. ${ }^{40}$

\section{Meta-biases}

In order to determine whether any reporting bias is present, a detailed search for unpublished studies will be conducted, for example, accessing any past conference proceedings in the last 10 years, and internet searches, 
with consistency between protocols and published studies examined, if protocols are available. Currently, no trial registry exists for measurement property studies; therefore, trial registries will not be searched.

\section{Confidence in cumulative evidence}

Overall strength and quality of evidence will be investigated using a modified GRADE approach as recommended by the COSMIN guidelines for systematic reviews. ${ }^{40}$ Four of the GRADE factors will be used: risk of bias, inconsistency, imprecision and indirectness with publication bias being omitted. This is due to the difficulty in assessing this factor due to no registry currently existing for measurement properties. ${ }^{40}$

\section{Patient and public involvement}

The research question in this study was developed following consultations and discussion within patient and public involvement meetings. Patients will not be involved in the analysis and data collection of the systematic review.

\section{Clinical implications of this study}

$\mathrm{CS}$ is complex in nature and can cause significant disability and pain for individuals. This in turn can impact on an individual's recovery and other factors such as return to work rate following injury. A timely and accurate diagnosis by clinicians will allow tailored management and better outcome, in which outcome measures can complement diagnosis. The definition of CS itself is challenging, with multiple symptoms and clinical presentations, for example, the onset of CS can often be attributed to tissue damage or nerve injury, but in some cases no peripheral pathology exists. ${ }^{45}$ Established measurement properties of outcome measures are needed in clinical practice and research in order to use the most appropriate tool. This systematic review will allow clinicians to make informed decisions on the most appropriate outcome measures when assessing CS, working towards a more standardised approach of CS assessment. This systematic review will inform both clinical practice and further research into this topic.

\section{ETHICS AND DISSEMINATION}

No research ethics is required for this systematic review due to no patient data being collated. Results of this review will be submitted to be published in a peer review journal and presented at conferences.

Contributors All authors formulated the focus of the systematic review. NM is a PhD student and DF (lead supervisor), AR and NH are supervisors. NM drafted the initial version of the protocol manuscript with DF, AR and NH all providing guidance on topic, methodology and analyses. All authors reviewed and commented on each draft of the protocol. All authors have approved the final manuscript.

Funding This work was supported by National Institute for Health Research Surgical Reconstruction and Microbiology Research Centre (NIHR SRMRC).

Competing interests None declared.

Patient consent for publication Not required.
Provenance and peer review Not commissioned; externally peer reviewed.

Open access This is an open access article distributed in accordance with the Creative Commons Attribution Non Commercial (CC BY-NC 4.0) license, which permits others to distribute, remix, adapt, build upon this work non-commercially, and license their derivative works on different terms, provided the original work is properly cited, appropriate credit is given, any changes made indicated, and the use is non-commercial. See: http://creativecommons.org/licenses/by-nc/4.0/.

\section{REFERENCES}

1. Dworkin RH, Turk DC, Farrar JT, et al. Core outcome measures for chronic pain clinical trials: IMMPACT recommendations. Pain 2005;113(1-2):9-19.

2. Litcher-Kelly L, Martino SA, Broderick JE, et al. A systematic review of measures used to assess chronic musculoskeletal pain in clinical and randomized controlled clinical trials. J Pain 2007;8:906-13.

3. Fillingim RB, Loeser JD, Baron R, et al. Assessment of Chronic Pain: Domains, Methods, and Mechanisms. J Pain 2016;17(9 Suppl):T10-T20.

4. Vardeh D, Mannion RJ, Woolf CJ. Toward a Mechanism-Based Approach to Pain Diagnosis. J Pain 2016;17:T50-T69.

5. WHO. World Health Organisation. International Classification of Functioning, Disability and Health. Geneva, Switzerland: World Health Organisation, 2001.

6. Woolf CJ. What is this thing called pain? $J$ Clin Invest 2010;120:3742-4.

7. Woolf CJ. Central sensitization: implications for the diagnosis and treatment of pain. Pain 2011;152:S2-S15.

8. Latremoliere A, Woolf CJ. Central sensitization: a generator of pain hypersensitivity by central neural plasticity. J Pain 2009;10:895-926.

9. Fingleton $\mathrm{C}$, Smart K, Moloney N, et al. Pain sensitization in people with knee osteoarthritis: a systematic review and meta-analysis. Osteoarthritis Cartilage 2015;23:1043-56.

10. Smart KM, Blake C, Staines A, et al. Mechanisms-based classifications of musculoskeletal pain: part 1 of 3 : symptoms and signs of central sensitisation in patients with low back $( \pm \mathrm{leg})$ pain. Man Ther 2012;17:336-44.

11. Van Oosterwijck J, Nijs J, Meeus M, et al. Evidence for central sensitization in chronic whiplash: a systematic literature review. Eur J Pain 2013;17:299-312.

12. Smart KM, Blake C, Staines A, et al. Self-reported pain severity, quality of life, disability, anxiety and depression in patients classified with 'nociceptive', 'peripheral neuropathic' and 'central sensitisation' pain. The discriminant validity of mechanisms-based classifications of low back ( \pm leg) pain. Man Ther 2012;17:119-25.

13. Imamura M, Imamura ST, Kaziyama $\mathrm{HH}$, et al. Impact of nervous system hyperalgesia on pain, disability, and quality of life in patients with knee osteoarthritis: a controlled analysis. Arthritis Rheum 2008;59:1424-31.

14. Artus M, Campbell P, Mallen CD, et al. Generic prognostic factors for musculoskeletal pain in primary care: a systematic review. BMJ Open 2017;7:e012901.

15. Goldsmith R, Wright C, Bell SF, et al. Cold hyperalgesia as a prognostic factor in whiplash associated disorders: a systematic review. Man Ther 2012;17:402-10.

16. Sterling M, Jull G, Vicenzino B, et al. Sensory hypersensitivity occurs soon after whiplash injury and is associated with poor recovery. Pain 2003;104:509-17.

17. Walton DM, Macdermid JC, Nielson W, et al. Pressure pain threshold testing demonstrates predictive ability in people with acute whiplash. J Orthop Sports Phys Ther 2011;41:658-65.

18. Rosenbloom BN, Khan S, McCartney C, et al. Systematic review of persistent pain and psychological outcomes following traumatic musculoskeletal injury. J Pain Res 2013;6:39.

19. Clay FJ, Watson WL, Newstead SV, et al. A systematic review of early prognostic factors for persisting pain following acute orthopedic trauma. Pain Res Manag 2012;17:35-44.

20. Rivara FP, Mackenzie EJ, Jurkovich GJ, et al. Prevalence of pain in patients 1 year after major trauma. Arch Surg 2008;143:282-7.

21. Rushton AB, Evans DW, Middlebrook N, et al. Development of a screening tool to predict the risk of chronic pain and disability following musculoskeletal trauma: protocol for a prospective observational study in the United Kingdom. BMJ Open 2018;8:e017876.

22. Geber C, Klein T, Azad S, et al. Test-retest and interobserver reliability of quantitative sensory testing according to the protocol of the German Research Network on Neuropathic Pain (DFNS): a multicentre study. Pain 2011;152:548-56. 
23. Rolke R, Baron R, Maier C, et al. Quantitative sensory testing in the German Research Network on Neuropathic Pain (DFNS): standardized protocol and reference values. Pain 2006;123:231-43.

24. Mayer TG, Neblett R, Cohen $\mathrm{H}$, et al. The development and psychometric validation of the central sensitization inventory. Pain Pract 2012;12:276-85.

25. Chesterton LS, Sim J, Wright CC, et al. Interrater reliability of algometry in measuring pressure pain thresholds in healthy humans, using multiple raters. Clin J Pain 2007;23:760-6.

26. Sim J, Wright C. Research in health care: concepts, designs and methods: Nelson Thornes. 2000.

27. Mokkink LB, Terwee CB, Patrick DL, et al. The COSMIN checklist for assessing the methodological quality of studies on measurement properties of health status measurement instruments: an international Delphi study, Qual Life Res 2010;19:539-49.

28. Mokkink LB, Terwee CB, Patrick DL, et al. The COSMIN study reached international consensus on taxonomy, terminology, and definitions of measurement properties for health-related patientreported outcomes. J Clin Epidemiol 2010;63:737-45.

29. Mokkink LB, de Vet HCW, Prinsen CAC, et al. COSMIN Risk of Bias checklist for systematic reviews of Patient-Reported Outcome Measures. Qual Life Res 2018;27:1171-9.

30. Mokkink LB, Terwee CB, Knol DL, et al. The COSMIN checklist for evaluating the methodological quality of studies on measurement properties: a clarification of its content. BMC Med Res Methodol 2010;10:22.

31. Clark J, Nijs J, Yeowell G, et al. What are the predictors of altered central pain modulation in chronic musculoskeletal pain populations? a systematic review. Pain Physician 2017;20:487.

32. Nijs J, Torres-Cueco R, van Wilgen CP, et al. Applying modern pain neuroscience in clinical practice: criteria for the classification of central sensitization pain. Pain Physician 2014;17:447-57.

33. Alqarni AM, Manlapaz D, Baxter D, Mohammad AA, Donald M, David $\mathrm{B}$, et al. Test Procedures to Assess Somatosensory Abnormalities in Individuals with Peripheral Joint Pain: A Systematic Review of Psychometric Properties. Pain Pract 2018;18.

34. Moloney NA, Hall TM, Doody CM. Reliability of thermal quantitative sensory testing: a systematic review. J Rehabil Res Dev 2012;49:191.
35. Moher D, Shamseer L, Clarke M, et al. Preferred reporting items for systematic review and meta-analysis protocols (PRISMA-P) 2015 statement. Syst Rev 2015;4:1.

36. Clay FJ, Newstead SV, McClure RJ. A systematic review of early prognostic factors for return to work following acute orthopaedic trauma. Injury 2010;41:787-803.

37. Freynhagen R, Baron R, Gockel U, et al. painDETECT: a new screening questionnaire to identify neuropathic components in patients with back pain. Curr Med Res Opin 2006;22:1911-20.

38. Dahm J, Ponsford J. Comparison of long-term outcomes following traumatic injury: what is the unique experience for those with brain injury compared with orthopaedic injury? Injury 2015;46:142-9.

39. Terwee CB, Jansma EP, Riphagen II, et al. Development of a methodological PubMed search filter for finding studies on measurement properties of measurement instruments. Qual Life Res 2009;18:1115-23.

40. Prinsen CAC, Mokkink LB, Bouter LM, et al. COSMIN guideline for systematic reviews of patient-reported outcome measures. Qual Life Res 2018;27:1147-57.

41. van Tulder M, Furlan A, Bombardier C, et al. Updated method guidelines for systematic reviews in the cochrane collaboration back review group. Spine 2003;28:1290-9.

42. Furlan AD, Pennick V, Bombardier C, et al. Editorial Board, Cochrane Back Review Group. 2009 updated method guidelines for systematic reviews in the Cochrane Back Review Group. Spine 2009;34:1929-41.

43. Mokkink LB, Terwee CB, Gibbons E, et al. Inter-rater agreement and reliability of the COSMIN (COnsensus-based Standards for the selection of health status Measurement Instruments) checklist. BMC Med Res Methodol 2010;10:82.

44. Terwee CB, Bot SD, de Boer MR, et al. Quality criteria were proposed for measurement properties of health status questionnaires. J Clin Epidemiol 2007;60:34-42.

45. Woolf CJ. Pain amplification-A perspective on the how, why, when, and where of central sensitization. J Appl Biobehav Res 2018;23:e12124. 